\title{
Limit Distributions of Eigenvalues for Random Block Toeplitz and Hankel Matrices
}

\author{
Yi-Ting Li, Dang-Zheng Liu and Zheng-Dong Wang \\ School of Mathematical Sciences \\ Peking University \\ Beijing, 100871, P. R. China
}

March 18, 2010

\begin{abstract}
Block Toeplitz and Hankel matrices arise in many aspects of applications. In this paper, we will research the distributions of eigenvalues for some models and get the semicircle law. Firstly we will give trace formulae of block Toeplitz and Hankel matrix. Then we will prove that the almost sure limit $\gamma_{T}^{(m)}\left(\gamma_{H}^{(m)}\right)$ of eigenvalue distributions of random block Toeplitz (Hankel) matrices exist and give the moments of the limit distributions where $m$ is the order of the blocks. Then we will prove the existence of almost sure limit of eigenvalue distributions of random block Toeplitz and Hankel band matrices and give the moments of the limit distributions. Finally we will prove that $\gamma_{T}^{(m)}\left(\gamma_{H}^{(m)}\right)$ converges weakly to the semicircle law as $m \rightarrow \infty$.
\end{abstract}

Key words: Random block Toeplitz matrix; Hankel matrix; Eigenvalues distribution; Band matrix; Semicircle law.

Mathematics Subject Classification (2000) 15A52

\section{Introduction}

In random matrix theory, a very important object is the eigenvalue distribution of a random matrix. If $A=\left(a_{i j}(\omega)\right)_{i, j=1}^{N}$ is a real symmetric random matrix where the $\left(a_{i j}(\omega)\right)$ 's are random variables on a probability space $\Omega$ with a probability measure $P$, then the eigenvalue distribution of $A$ is

$$
\mu_{A}=\frac{1}{N} \int_{\Omega} \sum_{j=1}^{N} \delta_{\lambda_{j}(\omega)} d P(\omega)
$$


where $\lambda_{j}(\omega)$ 's are the $N$ real eigenvalues of $A$.

The asymptotic behavior of the eigenvalue distribution is of much importance. In $[23,24]$, Wigner got the semicircle law for a wide class of real symmetric random matrices and this great result caused much development of random matrix theory. Recently in a review paper (see [1]), Bai proposed the study of random matrix models with certain additional linear structure. The properties of the distributions of eigenvalues for random Hankel and Toeplitz matrices with independent entries are listed among the unsolved random matrix problems posed in [1]. In [5], Bryc, Dembo and Jiang proved the existence of limit distribution $\gamma_{T}$ and $\gamma_{H}$ of real symmetric Toeplitz and Hankel matrices. The moments of $\gamma_{T}$ and $\gamma_{H}$ are the sum of volumes of solids. Hammond and Miller in [9] also proved the existence of $\gamma_{T}$ and $\gamma_{H}$ independently. In [15], Liu and Wang proved the existence of limit distribution $\gamma_{T}$ and $\gamma_{H}$ for real symmetric, complex Hermitian band Toeplitz and real symmetric band Hankel matrices. We notice that Basak and Bose (see [2]) and Kargin (see [13]) also did the same work independently. Especially, the limit distribution of random Toeplitz band matrices with bandwidth $b_{N}=o(N)$ is Gaussian.

Block Toeplitz and Hankel matrices arise in many aspects of mathematics, physics and technology (see $[8,10,14,19])$. A block Toeplitz matrix is a block matrix which can be written as

$$
T=\left(A_{i-j}\right)_{i, j=1}^{N}=\left(\begin{array}{ccccc}
A_{0} & A_{-1} & A_{-2} & \cdots & A_{-(N-1)} \\
A_{1} & A_{0} & A_{-1} & \cdots & A_{-(N-2)} \\
A_{2} & A_{1} & A_{0} & \cdots & A_{-(N-3)} \\
\vdots & \vdots & \vdots & \ddots & \vdots \\
A_{N-1} & A_{N-2} & A_{N-3} & \cdots & A_{0}
\end{array}\right)
$$

where $A_{s}=\left(a_{i j}(s)\right)_{i, j=1}^{m}$ is an $m \times m$ matrix, $\forall s \in\{-N+1, \ldots, N-1\}$. In [7], Gazzah, Regalia and Delmas researched the asymptotic behavior of the eigenvalue distribution for block Toeplitz matrices. In [18], Rashidi Far, Oraby, Bryc and Speicher have proved the existence of the limit distribution of eigenvalues for a random block Toeplitz matrix whose blocks are selfadjoint $m \times m$ matrices as $m \rightarrow \infty$, which implies that the double limit $\lim _{N \rightarrow \infty} \lim _{m \rightarrow \infty}$ gives the semicircle law. In this paper, we will study the limit distributions of real symmetric random block Toeplitz and Hankel matrices as $\lim _{N \rightarrow \infty}$ and $\lim _{m \rightarrow \infty} \lim _{N \rightarrow \infty}$.

In a block Toeplitz matrix with the form mentioned above, we suppose the $a_{i j}(s)$ 's are real random variables. For symmetry, we need $A_{s}=\left(A_{-s}\right)^{T}$. For independence of the elements, we suppose: 
(1) $a_{i_{1} j_{1}}\left(s_{1}\right)$ and $a_{i_{2} j_{2}}\left(s_{2}\right)$ are independent if $\left|s_{1}\right| \neq\left|s_{2}\right|$,

(2) If $s \neq 0$ and $\left(i_{1}, j_{1}\right) \neq\left(i_{2}, j_{2}\right)$ then $a_{i_{1} j_{1}}(s)$ and $a_{i_{2} j_{2}}(s)$ are independent,

(3) If $\left(i_{1}, j_{1}\right) \neq\left(i_{2}, j_{2}\right)$ and $\left(i_{1}, j_{1}\right) \neq\left(j_{2}, i_{2}\right)$ then $a_{i_{1} j_{1}}(0)$ and $a_{i_{2} j_{2}}(0)$ are independent.

In addition, we need the following uniform boundedness conditoin:

(4)

$$
E\left(a_{i j}(s)\right)=0, E\left(\left|a_{i j}(s)\right|^{2}\right)=1,-(N-1) \leq s \leq N-1,1 \leq i, j \leq m
$$

and

$$
\sup _{\substack{N \in \mathbb{N} \\-(N-1) \leq s \leq N-1}}\left\{\left|a_{i j}(s)\right|^{k} \mid 1 \leq i, j \leq m\right\}=C_{k, m}<+\infty
$$

and

$$
\sup _{m \in \mathbb{N}} C_{k, m}=C_{k}<+\infty .
$$

A block Hankel matrix is a block matrix which can be written as

$$
H=\left(A_{N+1-i-j}\right)_{i, j=1}^{N}=\left(\begin{array}{ccccc}
A_{N-1} & A_{N-2} & A_{N-3} & \cdots & A_{0} \\
A_{N-2} & A_{N-3} & A_{N-4} & \cdots & A_{-1} \\
A_{N-3} & A_{N-4} & A_{N-5} & \cdots & A_{-2} \\
\vdots & \vdots & \vdots & \ddots & \vdots \\
A_{0} & A_{-1} & A_{-2} & \cdots & A_{-(N-1)}
\end{array}\right)
$$

where $A_{s}=\left(a_{i j}(s)\right)_{i, j=1}^{m}$ is an $m \times m$ matrix, $\forall s \in\{-N+1, \ldots, N-1\}$. Similar to random block Toeplitz matrices, we suppose the $a_{i j}(s)$ 's are real random variables, $A_{s}=\left(A_{s}\right)^{T}$. In addition, we assume:

(1) $a_{i_{1} j_{1}}\left(s_{1}\right)$ and $a_{i_{2} j_{2}}\left(s_{2}\right)$ are independent if $s_{1} \neq s_{2}$,

(2) If $\left(i_{1}, j_{1}\right) \neq\left(i_{2}, j_{2}\right)$ and $\left(i_{1}, j_{1}\right) \neq\left(j_{2}, i_{2}\right)$ then $a_{i_{1} j_{1}}(s)$ and $a_{i_{2} j_{2}}(s)$ are independent,

(3)

$$
E\left(a_{i j}(s)\right)=0, E\left(\left|a_{i j}(s)\right|^{2}\right)=1,-(N-1) \leq s \leq N-1,1 \leq i, j \leq m
$$

and

$$
\sup _{\substack{N \in \mathbb{N} \\-(N-1) \leq s \leq N-1}}\left\{\left|a_{i j}(s)\right|^{k} \mid 1 \leq i, j \leq m\right\}=C_{k, m}<+\infty
$$

and

$$
\sup _{m \in \mathbb{N}} C_{k, m}=C_{k}<+\infty .
$$


We will firstly give trace formulae of block Toeplitz and Hankel matrices in Section 2. Using those trace formulae, we will prove that the almost sure limit distributions of block Toeplitz and Hankel matrices exist in Section 3. In that section we will also give the moments of the limit distributions. In Section 4, we will prove the existence of almost sure limit distributions of block Toeplitz and Hankel band matrices and give the moments of the limit distributions. In Section 5 we will prove that for block Toeplitz and Hankel matrices, the double limit $\lim _{m \rightarrow \infty} \lim _{N \rightarrow \infty}$ gives the semicircle law.

\section{Trace Formulae of Block Toeplitz and Hankel Matrices}

Definition 2.1. Let $T$ be an $m N \times m N$ matrix and consist of $N^{2}$ blocks. If $T$ has the form

$$
T=\left(A_{i, j}\right)_{i, j=1}^{N}=\left(\begin{array}{ccccc}
A_{0} & A_{-1} & A_{-2} & \cdots & A_{-(N-1)} \\
A_{1} & A_{0} & A_{-1} & \cdots & A_{-(N-2)} \\
A_{2} & A_{1} & A_{0} & \cdots & A_{-(N-3)} \\
\vdots & \vdots & \vdots & \ddots & \vdots \\
A_{N-1} & A_{N-2} & A_{N-3} & \cdots & A_{0}
\end{array}\right)
$$

where $\left\{A_{-(N-1)}, \ldots, A_{0}, \ldots, A_{N-1}\right\}$ is a set of $m \times m$ matrices and $A_{i, j}=$ $A_{i-j}=\left(a_{p q}(i-j)\right)_{p, q=1}^{m}$, then we call $T$ a block Toeplitz matrix.

Let $H$ be an $m N \times m N$ matrix and can be written as $H=\Phi T$ where $T$ is a block Toeplitz matrix and

$$
\Phi=\left(\begin{array}{cccc}
0 & \cdots & 0 & I_{m} \\
0 & \cdots & I_{m} & 0 \\
\vdots & \ddots & \vdots & \vdots \\
I_{m} & \cdots & 0 & 0
\end{array}\right)
$$

where $I_{m}$ is the $m \times m$ unit matrix. Then we call $H$ a block Hankel matrix.

For convenience, let $b_{N}=N-1$.

Lemma 2.2. Let $T=\left(A_{i-j}\right)_{i, j=1}^{N}$ be a block Toeplitz matrix and $A_{s}=$ $\left(a_{p q}(s)\right)_{p, q=1}^{m}$ where $-b_{N} \leq s \leq b_{N} ; 1 \leq p, q \leq m$. Then we have a trace 
formula

$$
\begin{aligned}
\operatorname{tr}\left(T^{k}\right) & =\sum_{i=1}^{N} \sum_{j_{1}, \ldots, j_{k}=-b_{N}}^{b_{N}} \operatorname{tr}\left(A_{j_{1}} \cdots A_{j_{k}}\right) \prod_{l=1}^{k} I_{[1, N]}\left(i+\sum_{q=1}^{l} j_{q}\right) \delta_{0, \sum_{q=1}^{k} j_{q}} \\
& =\sum_{i=1}^{N} \sum_{j_{1}, \ldots, j_{k}=-b_{N}}^{b_{N}} \sum_{t_{1}, \ldots, t_{k}=1}^{m} a_{t_{1} t_{2}}\left(j_{1}\right) \cdots a_{t_{k} t_{1}}\left(j_{k}\right) \prod_{l=1}^{k} I_{[1, N]}\left(i+\sum_{q=1}^{l} j_{q}\right) \delta_{0, \sum_{q=1}^{k} j_{q}}^{k} .
\end{aligned}
$$

Lemma 2.3. Let $H=\Phi T$ be a block Hankel matrix and $T=\left(A_{i-j}\right)_{i, j=1}^{N} ; A_{s}=$ $\left(a_{p q}(s)\right)_{p, q=1}^{m}$ where $-b_{N} \leq s \leq b_{N} ; 1 \leq p, q \leq m$. Then we have the trace formula

$$
\begin{aligned}
& \operatorname{tr}\left(H^{k}\right)= \\
& \left\{\begin{array}{lll}
\sum_{i=1}^{N} \sum_{j_{1}, \ldots, j_{k}=-b_{N}}^{b_{N}} \operatorname{tr}\left(A_{j_{1}} \cdots A_{j_{k}}\right) \prod_{l=1}^{k} I_{[1, N]}\left(i-\sum_{q=1}^{l}(-1)^{q} j_{q}\right) \delta_{0, \sum_{q=1}^{k}(-1)^{q} j_{q}} & k \text { even } \\
\sum_{i=1}^{N} \sum_{j_{1}, \ldots, j_{k}=-b_{N}}^{b_{N}} \operatorname{tr}\left(A_{j_{1}} \cdots A_{j_{k}}\right) \prod_{l=1}^{k} I_{[1, N]}\left(i-\sum_{q=1}^{l}(-1)^{q} j_{q}\right) \delta_{2 i-1-N, \sum_{q=1}^{k}(-1)^{q} j_{q}} & k \text { odd }
\end{array}\right.
\end{aligned}
$$

where

$$
\operatorname{tr}\left(A_{j_{1}} \cdots A_{j_{k}}\right)=\sum_{t_{1}, \ldots, t_{k}=1}^{m} a_{t_{1} t_{2}}\left(j_{1}\right) \cdots a_{t_{k} t_{1}}\left(j_{k}\right) .
$$

To prove the above lemmas, we consider Kronecker product of two matrices (see [12]). Let $A=\left(a_{i j}\right)$ be an $m \times n$ matrix and $B$ be a $p \times q$ matrix. The Kronecker product of $A$ and $B$ is an $m p \times n q$ matrix:

$$
A \otimes B=\left(\begin{array}{ccc}
a_{11} B & \cdots & a_{1 n} B \\
\vdots & \ddots & \vdots \\
a_{m 1} B & \cdots & a_{m n} B
\end{array}\right) .
$$

Proof of Lemma 2.2. Let $\mathrm{B}$ and $\mathrm{F}$ be two $N \times N$ matrices and

$$
B=\left(\delta_{i+1, j}\right)_{i, j=1}^{N}=\left(\begin{array}{cccccc}
0 & 1 & 0 & \cdots & 0 & 0 \\
0 & 0 & 1 & \cdots & 0 & 0 \\
0 & 0 & 0 & \cdots & 0 & 0 \\
\vdots & \vdots & \vdots & \ddots & \vdots & \vdots \\
0 & 0 & 0 & \cdots & 0 & 1 \\
0 & 0 & 0 & \cdots & 0 & 0
\end{array}\right), F=\left(\delta_{i, j+1}\right)_{i, j=1}^{N}=\left(\begin{array}{cccccc}
0 & 0 & 0 & \cdots & 0 & 0 \\
1 & 0 & 0 & \cdots & 0 & 0 \\
0 & 1 & 0 & \cdots & 0 & 0 \\
\vdots & \vdots & \vdots & \ddots & \vdots & \vdots \\
0 & 0 & 0 & \cdots & 0 & 0 \\
0 & 0 & 0 & \cdots & 1 & 0
\end{array}\right) .
$$


Then $T=\sum_{j=0}^{b_{N}} B^{j} \otimes A_{-j}+\sum_{j=1}^{b_{N}} F^{j} \otimes A_{j}$. Let $e_{1}^{i}$ be the $i$ th unit vector in $\mathbb{R}^{N}$ and $e_{2}^{j}$ be the $j$ th unit vector in $\mathbb{R}^{m}$, then we have

$$
B^{l} \otimes A_{-l}\left(e_{1}^{i} \otimes e_{2}^{j}\right)=\left(B^{l} e_{1}^{i}\right) \otimes\left(A_{-l} e_{2}^{j}\right)=I_{[1, N]}(i-l) e_{1}^{i-l} \otimes\left(A_{-l} e_{2}^{j}\right)
$$

and

$$
F^{l} \otimes A_{l}\left(e_{1}^{i} \otimes e_{2}^{j}\right)=\left(F^{l} e_{1}^{i}\right) \otimes\left(A_{l} e_{2}^{j}\right)=I_{[1, N]}(i+l) e_{1}^{i+l} \otimes\left(A_{l} e_{2}^{j}\right)
$$

thus

$$
\begin{aligned}
T\left(e_{1}^{i} \otimes e_{2}^{j}\right) & =\sum_{l=0}^{b_{N}} I_{[1, N]}(i-l) e_{1}^{i-l} \otimes\left(A_{-l} e_{2}^{j}\right)+\sum_{l=1}^{b_{N}} I_{[1, N]}(i+l) e_{1}^{i+l} \otimes\left(A_{l} e_{2}^{j}\right) \\
& =\sum_{l=-b_{N}}^{b_{N}} I_{[1, N]}(i+l) e_{1}^{i+l} \otimes\left(A_{l} e_{2}^{j}\right),
\end{aligned}
$$

so

$$
T^{k}\left(e_{1}^{i} \otimes e_{2}^{j}\right)=\sum_{l_{1}, \ldots, l_{k}=-b_{N}}^{b_{N}} \prod_{r=1}^{k} I_{[1, N]}\left(i+\sum_{q=1}^{r} l_{q}\right) e_{1}^{i+\sum_{q=1}^{r} l_{q}} \otimes\left(A_{l_{k}} \cdots A_{l_{1}} e_{2}^{j}\right) .
$$

As $\left\{e_{1}^{i} \otimes e_{2}^{j} \mid 1 \leq i \leq N ; 1 \leq j \leq m\right\}$ is a standard basis of $\mathbb{R}^{m N}$, we have the trace formula

$$
\begin{aligned}
& \operatorname{tr}\left(T^{k}\right) \\
= & \sum_{i=1}^{N} \sum_{j=1}^{m}\left(e_{1}^{i} \otimes e_{2}^{j}\right)^{T} T^{k}\left(e_{1}^{i} \otimes e_{2}^{j}\right) \\
= & \sum_{i=1}^{N} \sum_{l_{1}, \ldots, l_{k}=-b_{N}}^{b_{N}} \prod_{r=1}^{k} I_{[1, N]}\left(i+\sum_{q=1}^{r} l_{q}\right) \sum_{j=1}^{m}\left(\left(e_{1}^{i}\right)^{T} \otimes\left(e_{2}^{j}\right)^{T}\right) \cdot\left(e_{1}^{i+\sum_{q=1}^{r} l_{q}} \otimes\left(A_{l_{k}} \cdots A_{l_{1}} e_{2}^{j}\right)\right) \\
= & \sum_{i=1}^{N} \sum_{l_{1}, \ldots, l_{k}=-b_{N}}^{b_{N}} \prod_{r=1}^{k} I_{[1, N]}\left(i+\sum_{q=1}^{r} l_{q}\right) \delta \sum_{q=1}^{k} l_{q} \cdot \sum_{j=1}^{m}\left(\left(e_{2}^{j}\right)^{T} A_{l_{k}} \cdots A_{l_{1}} e_{2}^{j}\right) \\
= & \sum_{i=1}^{N} \sum_{l_{1}, \ldots, l_{k}=-b_{N}}^{b_{N}} \prod_{r=1}^{k} I_{[1, N]}\left(i+\sum_{q=1}^{r} l_{q}\right) \operatorname{tr}\left(A_{l_{k}} \cdots A_{l_{1}}\right) \delta \sum_{0, \sum_{q=1}^{k} l_{q}} \cdot
\end{aligned}
$$


For $\operatorname{tr}\left(A_{l_{k}} \cdots A_{l_{1}}\right)=\operatorname{tr}\left(A_{l_{1}} \cdots A_{l_{k}}\right)$, we get

$$
\operatorname{tr}\left(T^{k}\right)=\sum_{i=1}^{N} \sum_{j_{1}, \ldots, j_{k}=-b_{N}}^{b_{N}} \operatorname{tr}\left(A_{j_{1}} \cdots A_{j_{k}}\right) \prod_{l=1}^{k} I_{[1, N]}\left(i+\sum_{q=1}^{l} j_{q}\right) \delta_{0, \sum_{q=1}^{k} j_{q}} .
$$

Note that

$$
\operatorname{tr}\left(A_{l_{1}} \cdots A_{l_{k}}\right)=\sum_{t_{1}, \ldots, t_{k}=1}^{m} a_{t_{1} t_{2}}\left(l_{1}\right) \cdots a_{t_{k} t_{1}}\left(l_{k}\right)
$$

and we directly get

$\operatorname{tr}\left(T^{k}\right)=\sum_{i=1}^{N} \sum_{j_{1}, \ldots, j_{k}=-b_{N}}^{b_{N}} \sum_{t_{1}, \ldots, t_{k}=1}^{m} a_{t_{1} t_{2}}\left(j_{1}\right) \cdots a_{t_{k} t_{1}}\left(j_{k}\right) \prod_{l=1}^{k} I_{[1, N]}\left(i+\sum_{q=1}^{l} j_{q}\right) \delta \sum_{0, \sum_{q=1}^{k} j_{q}}$.

Proof of Lemma 2.3. $\Phi=P \otimes I_{m}$ where $I_{m}$ is the $m \times m$ unit matrix and $P$ is an $N \times N$ matrix and

$$
P=\left(\begin{array}{cccc}
0 & \cdots & 0 & 1 \\
0 & \cdots & 1 & 0 \\
\vdots & \ddots & \vdots & \vdots \\
1 & \cdots & 0 & 0
\end{array}\right)
$$

So if $A$ is an $N \times N$ matrix and $B$ is an $m \times m$ matrix, then we have $\Phi(A \otimes B)=\left(P \otimes I_{m}\right)(A \otimes B)=(P A) \otimes B$. Note that $P e_{1}^{i}=e_{1}^{N+1-i}$.

As in the proof of Lemma 2.2, we have

$\Phi T\left(e_{1}^{i} \otimes e_{2}^{j}\right)=\left(P \otimes I_{m}\right)\left(\sum_{l=-b_{N}}^{b_{N}} I_{[1, N]}(i+l) e_{1}^{i+l} \otimes\left(A^{l} e_{2}^{j}\right)\right)=\sum_{l=-b_{N}}^{b_{N}} I_{[1, N]}(i+l) e_{1}^{N+1-(i+l)} \otimes\left(A^{l} e_{2}^{j}\right)$,

and then

$$
\begin{aligned}
& H^{k}\left(e_{1}^{i} \otimes e_{2}^{j}\right)=
\end{aligned}
$$

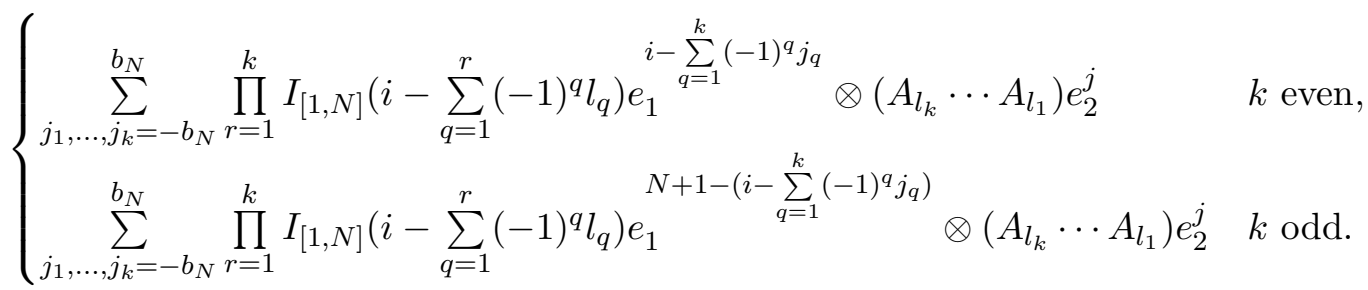


Thus we get

$$
\begin{aligned}
& \operatorname{tr}\left(H^{k}\right) \\
= & \sum_{i=1}^{N} \sum_{j=1}^{m}\left(e_{1}^{i} \otimes e_{2}^{j}\right)^{T} H^{k}\left(e_{1}^{i} \otimes e_{2}^{j}\right) \\
= & \begin{cases}\sum_{i=1}^{N} \sum_{j_{1}, \ldots, j_{k}=-b_{N}}^{b_{N}} \operatorname{tr}\left(A_{j_{1}} \cdots A_{j_{k}}\right) \prod_{l=1}^{k} I_{[1, N]}\left(i-\sum_{q=1}^{l}(-1)^{q} j_{q}\right) \delta \sum_{0=1}^{k}(-1)^{q} j_{q} & k \text { even } \\
\sum_{i=1}^{N} \sum_{j_{1}, \ldots, j_{k}=-b_{N}}^{b_{N}} \operatorname{tr}\left(A_{j_{1}} \cdots A_{j_{k}}\right) \prod_{l=1}^{k} I_{[1, N]}\left(i-\sum_{q=1}^{l}(-1)^{q} j_{q}\right) \delta \sum_{2 i-1-N, \sum_{q=1}^{k}(-1)^{q} j_{q}} & k \text { odd }\end{cases}
\end{aligned}
$$

where

$$
\operatorname{tr}\left(A_{j_{1}} \cdots A_{j_{k}}\right)=\sum_{t_{1}, \ldots, t_{k}=1}^{m} a_{t_{1} t_{2}}\left(j_{1}\right) \cdots a_{t_{k} t_{1}}\left(j_{k}\right) .
$$

\section{Limit Distributions of Random Block Toeplitz and Hankel Matrices}

Let $\sharp V$ denote the number of elements in an arbitrary finite set $V$. We now review the concept of partition (see $[15])$. Let $[n]=\{1,2, \ldots, n\}$.

(1) We call $\pi=\left\{V_{1}, V_{2}, \ldots, V_{r}\right\}$ a partition of $[n]$ if $\bigcup_{j=1}^{r} V_{j}=[n]$ and $V_{i} \cap V_{j}=$ $\emptyset$ if $i \neq j$.

(2) For $\forall p \in[n]$, we define $\pi(p)=i$ if $p \in V_{i}$. We write $p \sim_{\pi} q$ if $\pi(p)=\pi(q)$. (3) Let $\mathcal{P}(n)$ denote the set of all the partition of $[n]$. We define $\mathcal{P}_{2}(n)=$ $\left\{\pi=\left\{V_{1}, V_{2}, \ldots, V_{r}\right\} \in \mathcal{P}(n) \mid \sharp V_{i}=2, \forall i\right\}$ and $\mathcal{P}_{2}^{1}(n)=\left\{\pi=\left\{V_{1}, V_{2}, \ldots, V_{r}\right\} \in\right.$ $\mathcal{P}_{2}(n) \mid V_{i}$ has exactly one even element and one odd element, $\left.\forall i\right\}$.

Definition 3.1. Let $k \in \mathbb{N}, \pi=\left\{V_{1}, V_{2}, \ldots, V_{k}\right\} \in \mathcal{P}_{2}(2 k), V_{r}=\left\{a_{r}, b_{r}\right\}(1 \leq$ $r \leq k)$, then $\pi$ determines a system of linear equations with unknown variables $t_{1}, t_{2}, \ldots, t_{2 k}\left(\right.$ set $\left.t_{2 k+1}=t_{1}\right)$ :

$$
\begin{cases}t_{a_{i}}=t_{b_{i}+1} & 1 \leq i \leq k \\ t_{b_{i}}=t_{a_{i}+1} & 1 \leq i \leq k\end{cases}
$$

The number of linearly independent equations of this system is denoted by $f(\pi)$. Clearly we have $0 \leq f(\pi) \leq 2 k$. 
Theorem 3.2. Let $T_{N}=\left(A_{i-j}\right)_{i, j=1}^{N}$ be an $m N \times m N$ random block Toeplitz matrix where $A_{s}=\left(a_{i j}(s)\right)_{i, j=1}^{m}, A_{-s}=\left(A_{s}\right)^{T}$, and $a_{i j}(s)$ 's are random variables satisfying the four conditions mentioned in introduction. Let $X_{N}=$ $\frac{T_{N}}{\sqrt{m N}}$, then eigenvalue distribution $\mu_{X_{N}}$ converges almost surely to a symmetric probability distribution $\gamma_{T}^{(m)}$ which is determined by its even moments

$m_{2 k}\left(\gamma_{T}^{(m)}\right)=\sum_{\pi \in \mathcal{P}_{2}(2 k)} m^{k-1-f(\pi)} \int_{[0,1] \times[-1,1]} \prod_{j=1}^{2 k} I_{[0,1]}\left(x_{0}+\sum_{q=1}^{j} \epsilon_{\pi}(q) x_{\pi(q)}\right) \prod_{l=0}^{k} \mathrm{~d} x_{l}$

where

$$
\epsilon_{\pi}(q)= \begin{cases}1 & \text { if } q \text { is the smaller element of } V_{\pi(q)}, \\ -1 & \text { if } q \text { is the larger element of } V_{\pi(q)} .\end{cases}
$$

Proof of Theorem 3.2. The $k$ th moment of $\mu_{X_{N}}$ is given by

$$
m_{k, N}=\int x^{k} \mathrm{~d} \mu_{X_{N}}=\frac{1}{m N} E\left(\operatorname{tr} X_{N}^{k}\right)=\frac{1}{m N}(m N)^{-\frac{k}{2}} E\left(\operatorname{tr} T_{N}^{k}\right) .
$$

Using Lemma 2.2, we get

$m_{k, N}=\frac{1}{m N}(m N)^{-\frac{k}{2}} \sum_{i=1}^{N} \sum_{j_{1}, \ldots, j_{k}=-b_{N}}^{b_{N}} \sum_{t_{1}, \ldots, t_{k}=1}^{m} E\left(a_{t_{1} t_{2}}\left(j_{1}\right) \cdots a_{t_{k} t_{1}}\left(j_{k}\right)\right) \prod_{l=1}^{k} I_{[1, N]}\left(i+\sum_{q=1}^{l} j_{q}\right) \delta \sum_{0, \sum_{q=1}^{k} j_{q}}$.

We will calculate the limit $\lim _{N \rightarrow \infty} m_{k, N}$.

Observe that

$$
\left|E\left(a_{t_{1} t_{2}}\left(j_{1}\right) \cdots a_{t_{k} t_{1}}\left(j_{k}\right)\right)\right| \leq D_{k} \quad\left(\forall j_{1}, \ldots, j_{k}, t_{1}, \ldots, t_{k}\right),
$$

where $D_{k}=\left(\max \left\{C_{1}, \ldots, C_{k}\right\}\right)^{k}$. If $\left|j_{p}\right| \neq\left|j_{q}\right|$, then $a_{t_{p} t_{p+1}}\left(j_{p}\right)$ and $a_{t_{q} t_{q+1}}\left(j_{q}\right)$ are independent. Let $p \in\{1,2, \ldots, k\}$. By the independence conditions and $E\left(a_{i j}(s)\right)=0$, we observe that if $E\left(a_{t_{1} t_{2}}\left(j_{1}\right) \cdots a_{t_{k} t_{1}}\left(j_{k}\right)\right) \neq 0$, then for any $p \in\{1, \ldots, k\}, \exists q \in\{1, \ldots, k\}, q \neq p$, s.t. $\left|j_{q}\right|=\left|j_{p}\right|$. So there are at most $\left[\frac{k}{2}\right]$ different elements in $V=\left\{\left|j_{1}\right|, \ldots,\left|j_{k}\right|\right\}$. Further, we have

$$
\begin{aligned}
\left|\sum_{j_{1}, \ldots, j_{k}=-b_{N}}^{b_{N}} E\left(a_{t_{1} t_{2}}\left(j_{1}\right) \cdots a_{t_{k} t_{1}}\left(j_{k}\right)\right)\right| & =\left|\sum_{s=1}^{\left[\frac{k}{2}\right]} \sum_{\sharp V=s} E\left(a_{t_{1} t_{2}}\left(j_{1}\right) \cdots a_{t_{k} t_{1}}\left(j_{k}\right)\right)\right| \\
& \leq \sum_{s=1}^{\left[\frac{k}{2}\right]} \eta_{k, s} \frac{\left(b_{N}+1\right) !}{\left(b_{N}+1-s\right) !} 2^{k} D_{k}
\end{aligned}
$$


where $\eta_{k, s}=\sharp\left\{\pi=\left\{U_{1}, \ldots, U_{s}\right\} \in \mathcal{P}(k) \mid \sharp U_{i} \geq 2, \forall i\right\}$.

So $\left|\sum_{j_{1}, \ldots, j_{k}=-b_{N}}^{b_{N}} E\left(a_{t_{1} t_{2}}\left(j_{1}\right) \cdots a_{t_{k} t_{1}}\left(j_{k}\right)\right)\right|=O\left(N^{\left[\frac{k}{2}\right]}\right)$ and then $m_{k, N}=O\left(N^{\left[\frac{k}{2}\right]-\frac{k}{2}}\right)$.

Thus for odd $k, m_{k, N}=o(1)$. Now we only have to consider $m_{2 k, N}$.

$\pi$ is a partition of $[2 k]=\{1,2, \ldots, 2 k\}$ and $p \sim_{\pi} q \Longleftrightarrow\left|j_{p}\right|=\left|j_{q}\right|$, then

we have

$$
\begin{aligned}
& \sum_{i=1}^{N} \sum_{\pi \in \mathcal{P}(2 k)}^{m_{2 k, N}} \sum_{\substack{j_{1}, \ldots, j_{2 k}=-b_{N} \\
p \sim \pi q,}}^{b_{N}} \sum_{\substack{j_{p}|=| j_{q} \mid \\
t_{1}, \ldots, t_{2 k}=1}}^{m} \frac{E\left(a_{t_{1} t_{2}}\left(j_{1}\right) \cdots a_{t_{2 k} t_{1}}\left(j_{2 k}\right)\right)}{(m N)^{k+1}} \prod_{l=1}^{2 k} I_{[1, N]}\left(i+\sum_{q=1}^{l} j_{q}\right) \delta_{0, \sum_{q=1}^{2 k} j_{q}} \\
+ & o(1) .
\end{aligned}
$$

Again by the assumptions in the introduction, the contribution of the partitions which are not pair partition to $m_{2 k, N}$ is $o(1)$. So we only have to consider the pair partitions. Suppose $\pi \in \mathcal{P}_{2}(2 k)$. If $p \sim_{\pi} q$, then $j_{p}=j_{q}$ or $j_{p}=-j_{q}$. Under the condition $\sum_{q=1}^{2 k} j_{q}=0$ according to (3.3), considering the main contribution to the trace, we should take $j_{p}=-j_{q}$. Otherwise there exists $p_{0}, q_{0} \in[2 k]$ such that

$$
j_{p_{0}}=j_{q_{0}}=\frac{1}{2}\left(j_{p_{0}}+j_{q_{0}}-\sum_{q=1}^{2 k} j_{q}\right) .
$$

We can choose other $k-1$ distinct numbers, which determine $j_{p_{0}}=j_{q_{0}}$ and then there is a loss of at least one degree of freedom and the contribution of such terms is $O\left(N^{-1}\right)$. Therefore we have

$$
\begin{aligned}
& m_{2 k, N} \\
& =\sum_{i=1}^{N} \sum_{\pi \in \mathcal{P}_{2}(2 k)} \sum_{\substack{j_{1}, \ldots, j_{2 k}=-b_{N} \\
p \sim \pi q}}^{b_{N}} \sum_{t_{p}, \ldots, t_{2 k}=-1}^{m} \frac{E\left(a_{t_{1} t_{2}}\left(j_{1}\right) \cdots a_{t_{2 k} t_{1}}\left(j_{2 k}\right)\right)}{(m N)^{k+1}} \prod_{l=1}^{2 k} I_{[1, N]}\left(i+\sum_{q=1}^{l} j_{q}\right) \\
& +\quad o(1)
\end{aligned}
$$

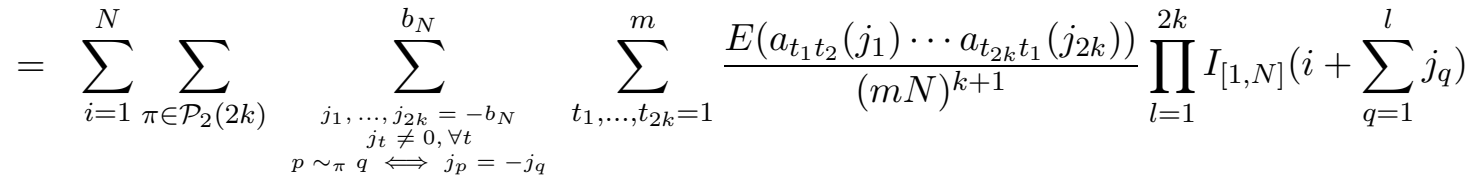

$$
\begin{aligned}
& +o(1) \text {. }
\end{aligned}
$$


Now we have to calculate

$$
\sum_{t_{1}, \ldots, t_{2 k}=1}^{m} E\left(a_{t_{1} t_{2}}\left(j_{1}\right) \cdots a_{t_{2 k} t_{1}}\left(j_{2 k}\right)\right)
$$

with the assumption that $j_{t} \neq 0, \forall t$. For convenience we let $t_{2 k+1}=t_{1}$. Suppose

$$
E\left(a_{t_{1} t_{2}}\left(j_{1}\right) \cdots a_{t_{2 k} t_{1}}\left(j_{2 k}\right)\right) \neq 0,
$$

then for any $p \sim_{\pi} q$ we have $a_{t_{p} t_{p+1}}\left(j_{p}\right)=a_{t_{q} t_{q+1}}\left(j_{q}\right)$. Suppose $p<q$.

$$
\begin{aligned}
& E\left(a_{t_{1} t_{2}}\left(j_{1}\right) \cdots a_{t_{2 k} t_{1}}\left(j_{2 k}\right)\right) \\
= & E\left(a_{t_{p} t_{p+1}}\left(j_{p}\right) a_{t_{q} t_{q+1}}\left(j_{q}\right)\right) \cdot E\left(a_{t_{1} t_{2}}\left(j_{1}\right) \cdots a_{t_{p} t_{p+1}}\left(j_{p}\right) \cdots a_{t_{q} t_{q+1}}\left(j_{q}\right) \cdots a_{t_{2 k} t_{1}}\left(j_{2 k}\right)\right) \\
= & 1 \cdot E\left(a_{t_{1} t_{2}}\left(j_{1}\right) \cdots a_{t_{p} t_{p+1}}\left(j_{p}\right) \cdots a_{t_{q} t_{q+1}}\left(j_{q}\right) \cdots a_{t_{2 k} t_{1}}\left(j_{2 k}\right)\right)
\end{aligned}
$$

where ${ }^{\wedge}$ marks omitted index. Note that $j_{p}=-j_{q}, A_{j_{p}}=\left(A_{j_{q}}\right)^{T}$ and $A_{j_{p}} \neq$ $A_{j_{q}}$ because $j_{p}=-j_{q} \neq 0 . a_{t_{p} t_{p+1}}\left(j_{p}\right)$ lies in the $t_{p}$-th row and the $t_{p+1}$-th column of $A_{j_{p}}$ and $a_{t_{q} t_{q+1}}\left(j_{q}\right)$ lies in the $t_{q}$-th row and the $t_{q+1}$-th column of $A_{j_{q}}$. So $t_{p}=t_{q+1}, t_{q}=t_{p+1}$. As $\pi=\left\{V_{1}, V_{2}, \ldots, V_{r}\right\}$ where $V_{i}=\left\{a_{i}, b_{i}\right\}(1 \leq$ $i \leq r)$, we have a system of equations:

$$
\left\{\begin{array}{l}
t_{a_{1}}=t_{b_{1}+1} \\
t_{a_{1}+1}=t_{b_{1}} \\
\cdots \\
t_{a_{k}}=t_{b_{k}+1} \\
t_{a_{k}+1}=t_{b_{k}}
\end{array} .\right.
$$

By Definition 3.1, there are $f(\pi)$ linearly independent equations in this system. So there are $2 k-f(\pi)$ variables taking values freely in $\{1,2, \ldots, m\}$ and the number of solutions of this system of equations is $m^{2 k-f(\pi)}$. In other words, there are $m^{2 k-f(\pi)}$ different $\left(t_{1}, \ldots, t_{2 k}\right)$ 's such that

$$
E\left(a_{t_{1} t_{2}}\left(j_{1}\right) \cdots a_{t_{2 k} t_{1}}\left(j_{2 k}\right)\right) \neq 0
$$

and this implies

$$
E\left(a_{t_{1} t_{2}}\left(j_{1}\right) \cdots a_{t_{2 k} t_{1}}\left(j_{2 k}\right)\right)=1 \text {. }
$$

So

$$
\sum_{t_{1}, \ldots, t_{2 k}=1}^{m} E\left(a_{t_{1} t_{2}}\left(j_{1}\right) \cdots a_{t_{2 k} t_{1}}\left(j_{2 k}\right)\right)=m^{2 k-f(\pi)} .
$$


Thus we have

$$
\begin{aligned}
& m_{2 k, N} \\
& =\frac{1}{(m N)^{k+1}} \sum_{i=1}^{N} \sum_{\pi \in \mathcal{P}_{2}(2 k)} m^{2 k-f(\pi)} \sum_{\substack{j_{1}, \ldots, j_{2 k}=-b_{N} \\
j \neq 0, \forall t \\
j \sim \pi}}^{b_{N} \stackrel{b_{N}}{\Longleftrightarrow} \prod_{p=-j_{q}}} \prod_{l=1}^{2 k} I_{[1, N]}\left(i+\sum_{q=1}^{l} j_{q}\right) \\
& +o(1) \\
& =\frac{1}{(m N)^{k+1}} \sum_{i=1}^{N} \sum_{\pi \in \mathcal{P}_{2}(2 k)} m^{2 k-f(\pi)} \sum_{\substack{j_{1}, \ldots, j_{2 k}=-b_{N} \\
p \sim \pi q}}^{b_{N}} \prod_{l=1}^{2 k} I_{[1, N]}\left(i+\sum_{q=1}^{l} j_{q}\right) \\
& +o(1) .
\end{aligned}
$$

Now for any $r \in\{1, \ldots, k\}$, let $x_{r}=j_{a_{r}}$, then $j_{q}=\left\{\begin{array}{ll}x_{r} & \text { if } q=a_{r} \\ -x_{r} & \text { if } q=b_{r}\end{array}\right.$. Remember Eq.(3.2), and then we have $j_{q}=\left\{\begin{array}{ll}x_{\pi(q)} & \text { if } \epsilon_{\pi}(q)=1 \\ -x_{\pi(q)} & \text { if } \epsilon_{\pi}(q)=-1\end{array}\right.$, so $j_{q}=\epsilon_{\pi}(q) x_{\pi(q)}$ and $m_{2 k, N}=(m N)^{-k-1} \sum_{i=1}^{N} \sum_{\pi \in \mathcal{P}_{2}(2 k)} \sum_{x_{1}, \ldots, x_{k}=-b_{N}}^{b_{N}} m^{2 k-f(\pi)} \prod_{l=1}^{2 k} I_{[1, N]}\left(i+\sum_{q=1}^{l} \epsilon_{\pi}(q) x_{\pi(q)}\right)+o(1)$, thus

$$
\begin{aligned}
\lim _{N \rightarrow \infty} m_{2 k, N} & =\sum_{\pi \in \mathcal{P}_{2}(2 k)} m^{k-1-f(\pi)} \int_{[0,1] \times[-1,1]^{k}} \prod_{j=1}^{2 k} I_{[0,1]}\left(x_{0}+\sum_{q=1}^{j} \epsilon_{\pi}(q) x_{\pi(q)}\right) \prod_{l=0}^{k} \mathrm{~d} x_{l} . \\
\text { Let } m_{2 k} & =\sum_{\pi \in \mathcal{P}_{2}(2 k)} m^{k-1-f(\pi)} \int_{[0,1] \times[-1,1]^{k}} \prod_{j=1}^{2 k} I_{[0,1]}\left(x_{0}+\sum_{q=1}^{l} \epsilon_{\pi}(q) x_{\pi(q)}\right) \prod_{l=0}^{k} \mathrm{~d} x_{l}
\end{aligned}
$$

and $m_{2 k-1}=0(k \in \mathbb{N})$, then for any $k \in \mathbb{N}$ we have $\lim _{N \rightarrow \infty} m_{k, N}=m_{k}$. It is easy to see that $m_{2 k} \leq \sum_{\pi \in \mathcal{P}_{2}(2 k)} m^{k-1-f(\pi)} \leq(2 k-1) ! ! \cdot m^{k-1}$ and then using Carleman's theorem (see [6]) we know that the limit distribution $\gamma_{T}^{(m)}$ is uniquely determined by its moments $\left\{m_{k}\right\}_{k=0}^{\infty}$.

Now we prove the almost sure convergence. It is sufficient to prove

$$
\sum_{N=1}^{\infty} \frac{1}{N^{4}} E\left(\left(\operatorname{tr} X_{N}^{k}-E\left(\operatorname{tr} X_{N}^{k}\right)\right)^{4}\right)<\infty
$$




$$
\begin{aligned}
& \operatorname{tr} X_{N}^{k}=(m N)^{-\frac{k}{2}} \operatorname{tr} T_{N}^{k} \\
& =(m N)^{-\frac{k}{2}} \sum_{i=1}^{N} \sum_{t_{1}, \ldots, t_{k}=1}^{m} \sum_{j_{1}, \ldots, j_{k}=-b_{N}}^{b_{N}} a_{t_{1} t_{2}}\left(j_{1}\right) \cdots a_{t_{k} t_{1}}\left(j_{k}\right) \prod_{l=1}^{k} I_{[1, N]}\left(i+\sum_{q=1}^{l} j_{q}\right) \delta \sum_{0, \sum_{q=1}^{k} j_{q}} .
\end{aligned}
$$

For convenience we let

$$
\begin{array}{r}
\mathbf{j}=\left(j_{1}, \ldots, j_{k}\right), \quad \mathbf{t}=\left(t_{1}, \ldots, t_{k}\right), \\
A[i, \mathbf{t}, \mathbf{j}]=a_{t_{1} t_{2}}\left(j_{1}\right) \cdots a_{t_{k} t_{1}}\left(j_{k}\right) \prod_{l=1}^{k} I_{[1, N]}\left(i+\sum_{q=1}^{l} j_{q}\right) \delta_{0, \sum_{q=1}^{k} j_{q}}
\end{array}
$$

and we use $\sum_{i, \mathbf{t}, \mathbf{j}}$ to denote $\sum_{i=1}^{N} \sum_{t_{1}, \ldots, t_{k}=1}^{m} \sum_{j_{1}, \ldots, j_{k}=-b_{N}}^{b_{N}}$, then we have

$$
\begin{aligned}
& \frac{1}{N^{4}} E\left(\left(\operatorname{tr} X_{N}^{k}-E\left(\operatorname{tr} X_{N}^{k}\right)\right)^{4}\right) \\
= & \frac{1}{N^{4}} E\left((m N)^{-\frac{k}{2}} \sum_{i, \mathbf{t}, \mathbf{j}} A[i, \mathbf{t}, \mathbf{j}]-E\left((m N)^{-\frac{k}{2}} \sum_{i, \mathbf{t}, \mathbf{j}} A[i, \mathbf{t}, \mathbf{j}]\right)\right)^{4} \\
= & \frac{1}{N^{2 k+4} m^{2 k}} E\left(\sum_{i, \mathbf{t}, \mathbf{j}}(A[i, \mathbf{t}, \mathbf{j}]-E A[i, \mathbf{t}, \mathbf{j}])\right)^{4} \\
= & \frac{1}{N^{2 k+4} m^{2 k}} E\left(\sum_{v=1}^{4} \sum_{i^{v}, \mathbf{t}^{v}, \mathbf{j}^{v}} \prod_{v=1}^{4}\left(A\left[i^{v}, \mathbf{t}^{v}, \mathbf{j}^{v}\right]-E A\left[i^{v}, \mathbf{t}^{v}, \mathbf{j}^{v}\right]\right)\right)
\end{aligned}
$$

where $\sum_{i^{v}, \mathbf{t}^{v}, \mathbf{j}^{v}}=\sum_{i^{v}=1}^{N} \sum_{t_{1}^{v}, \ldots, t_{k}^{v}=1}^{m} \sum_{j_{1}^{v}, \ldots, j_{k}^{v}=-b_{N}}^{b_{N}}$. let

For given $\mathbf{j}^{1}=\left(j_{1}^{1}, \ldots, j_{k}^{1}\right), \mathbf{j}^{2}=\left(j_{1}^{2}, \ldots, j_{k}^{2}\right), \mathbf{j}^{3}=\left(j_{1}^{3}, \ldots, j_{k}^{3}\right), \mathbf{j}^{4}=\left(j_{1}^{4}, \ldots, j_{k}^{4}\right)$,

$$
\mathbf{J}=\left(j_{1}^{1}, \ldots, j_{k}^{1}, \ldots, j_{1}^{4}, \ldots, j_{k}^{4}\right) \in\left\{-b_{N}, \ldots, b_{N}\right\}^{4 k} .
$$

Set $S^{\mathbf{J}}=\left\{\left|j_{1}^{1}\right|, \ldots,\left|j_{k}^{1}\right|, \ldots,\left|j_{1}^{4}\right|, \ldots,\left|j_{k}^{4}\right|\right\}$. We use $p(\mathbf{J})$ to denote the number of different elements of $S^{\mathbf{J}}$.

We construct a set of numbers with multiplicities $S_{\mathbf{J}}=\left\{j_{1}^{1}, \ldots, j_{k}^{1}, \ldots, j_{1}^{4}, \ldots, j_{k}^{4}\right\}$. Please note that if $\left|j_{u_{1}}^{v_{1}}\right|=\left|j_{u_{2}}^{v_{2}}\right|=a$, then $a$ appears twice in $S_{\mathbf{J}}$. Let $S_{1}, \ldots, S_{p(\mathbf{J})}$ be subsets of $S_{\mathbf{J}}$ such that:

(a) for all $w$, the elements of $S_{w}$ have the same absolute value;

(b) if $w_{1} \neq w_{2}$, then the absolute value of the elements in $S_{w_{1}}$ is different from the absolute value of the elements in $S_{w_{2}}$; 
(c) $\bigcup_{r=1}^{p} S_{r}=S_{\mathbf{J}}$. It is easy to see that $S_{1}, \ldots, S_{p(\mathbf{J})}$ are uniquely determined by $\mathbf{J}$. Then we have

$$
\left\{-b_{N}, \ldots, b_{N}\right\}^{4 k}=W_{1} \cup W_{2} \cup W_{3}
$$

where

$$
\begin{gathered}
W_{1}=\left\{\mathbf{J} \in\left\{-b_{N}, \ldots, b_{N}\right\}^{4 k} \mid p(\mathbf{J}) \leq 2 k-2\right\}, \\
W_{2}=\left\{\mathbf{J} \in\left\{-b_{N}, \ldots, b_{N}\right\}^{4 k} \mid p(\mathbf{J})=2 k-1\right\}, \\
W_{3}=\left\{\mathbf{J} \in\left\{-b_{N}, \ldots, b_{N}\right\}^{4 k} \mid p(\mathbf{J})=2 k\right\} .
\end{gathered}
$$

Therefore we have

$$
\begin{aligned}
& \frac{1}{N^{4}} E\left(\left(\operatorname{tr} X_{N}^{k}-E\left(\operatorname{tr} X_{N}^{k}\right)\right)^{4}\right) \\
& =\frac{1}{N^{2 k+4} m^{2 k}} E\left(\sum_{\substack{i^{1}, i^{2}, i^{3}, i^{4} \\
\mathbf{t}^{1}, \mathbf{t}^{2}, \mathbf{t}^{3}, \mathbf{t}^{4}}} \sum_{\mathbf{J} \in W_{1}} \prod_{v=1}^{4}\left(A\left[i^{v}, \mathbf{t}^{v}, \mathbf{j}^{v}\right]-E A\left[i^{v}, \mathbf{t}^{v}, \mathbf{j}^{v}\right]\right)\right) \\
& +\frac{1}{N^{2 k+4} m^{2 k}} E\left(\sum_{\substack{i^{1}, i^{2}, i^{3}, i^{4} \\
\mathbf{t}^{1}, \mathbf{t}^{2}, \mathbf{t}^{3}, \mathbf{t}^{4}}} \sum_{\mathbf{J} \in W_{2}} \prod_{v=1}^{4}\left(A\left[i^{v}, \mathbf{t}^{v}, \mathbf{j}^{v}\right]-E A\left[i^{v}, \mathbf{t}^{v}, \mathbf{j}^{v}\right]\right)\right) \\
& +\frac{1}{N^{2 k+4} m^{2 k}} E\left(\sum_{\substack{i^{1}, i^{2}, i^{3}, i^{4} \\
\mathbf{t}^{1}, \mathbf{t}^{2}, \mathbf{t}^{3}, \mathbf{t}^{4}}} \sum_{\mathbf{J} \in W_{3}} \prod_{v=1}^{4}\left(A\left[i^{v}, \mathbf{t}^{v}, \mathbf{j}^{v}\right]-E A\left[i^{v}, \mathbf{t}^{v}, \mathbf{j}^{v}\right]\right)\right) \\
& =\Phi_{1}+\Phi_{2}+\Phi_{3} \text {. }
\end{aligned}
$$

For given $j_{1}^{1}, \ldots, j_{k}^{4}, t_{1}^{1}, \ldots, t_{k}^{4}, i^{1}, \ldots, i^{4}$, suppose $E\left(\prod_{v=1}^{4}\left(A\left[i^{v}, \mathbf{t}^{v}, \mathbf{j}^{v}\right]-E A\left[i^{v}, \mathbf{t}^{v}, \mathbf{j}^{v}\right]\right)\right) \neq$ 0 . Then $\sum_{i=1}^{k} j_{i}^{v}=0(1 \leq v \leq 4)$ and from independence conditions we know that:

$$
\forall j_{u}^{v}, \exists\left(u_{1}, v_{1}\right) \neq(u, v) \text { s.t. }\left|j_{u}^{v}\right|=\left|j_{u_{1}}^{v_{1}}\right|
$$

and that

$$
\forall v_{1}, \exists v_{2} \neq v_{1} \text { and } u_{1}, u_{2} \text { s.t. }\left|j_{u_{1}}^{v_{1}}\right|=\left|j_{u_{2}}^{v_{2}}\right|
$$


otherwise,

$$
\begin{aligned}
& E\left(\prod_{v=1}^{4}\left(A\left[i^{v}, \mathbf{t}^{v}, \mathbf{j}^{v}\right]-E A\left[i^{v}, \mathbf{t}^{v}, \mathbf{j}^{v}\right]\right)\right) \\
= & E\left(\left(A\left[i^{v_{1}}, \mathbf{t}^{v_{1}}, \mathbf{j}^{v_{1}}\right]-E A\left[i^{v_{1}}, \mathbf{t}^{v_{1}}, \mathbf{j}^{v_{1}}\right]\right)\right) \cdot E\left(\prod_{\substack{1 \leq v \leq 4 \\
v \neq v_{1}}}\left(A\left[i^{v}, \mathbf{t}^{v}, \mathbf{j}^{v}\right]-E A\left[i^{v}, \mathbf{t}^{v}, \mathbf{j}^{v}\right]\right)\right) \\
= & 0 .
\end{aligned}
$$

We now evaluate $\Phi_{1}, \Phi_{2}$ and $\Phi_{3}$.

Evaluation of $\Phi_{1}$. Suppose $p \in \mathbb{N}$ and $p \leq 2 k-2$. There are at most $R_{k, p} \cdot\left(b_{N}+1\right)^{p} \cdot 2^{4 k}$ different $\mathbf{J}$ 's that satisfy $p(\mathbf{J})=p$, where $R_{k, p}=\sharp\left\{\pi=\left\{U_{1}, . ., U_{p}\right\} \mid \pi \in \mathcal{P}(4 k)\right\}$. So we have

$$
\begin{aligned}
\Phi_{1} & \leq \sum_{p=1}^{2 k-2} \frac{1}{N^{2 k+4} m^{2 k}} \cdot N^{4} \cdot m^{4 k} \cdot R_{k, p} \cdot\left(b_{N}+1\right)^{p} \cdot 2^{4 k} \cdot M \\
& \leq C \cdot b_{N}^{-2}
\end{aligned}
$$

where

$$
M=\sup _{N}\left\{\max _{\substack{1 \leq i^{v} \leq N \\ \mathbf{J} \in\left\{-b_{N}, \ldots, b_{N}\right\}^{4 k} \\ \mathbf{t}^{v} \in\{1, \ldots, m\}^{k}}}\left|E\left[\prod_{v=1}^{4}\left(A\left[i^{v}, \mathbf{t}^{v}, \mathbf{j}^{v}\right]-E A\left[i^{v}, \mathbf{t}^{v}, \mathbf{j}^{v}\right]\right)\right]\right|\right\}
$$

and $C$ is independent of $N$. From (1.1), (1.2) and (1.3), we know that such $M$ exists.

Evaluation of $\Phi_{2}$. Suppose $\mathbf{J} \in W_{2}$. For any $v \in\{1,2,3,4\}$, if $\exists u \in\{1,2, \ldots, k\}$ such that $\forall u_{1} \neq u,\left|j_{u}^{v}\right| \neq\left|j_{u_{1}}^{v}\right|$, then $j_{u}^{v}$ and its absolute value are determined by $j_{1}^{v}, \ldots, j_{u-1}^{v}, j_{u+1}^{v}, \ldots, j_{k}^{v}$ for $\sum_{i=1}^{k} j_{i}^{v}=0$. Thus

$$
\begin{aligned}
& \sharp\left\{\mathbf{J}=\left(j_{1}^{1}, \ldots, j_{k}^{4}\right) \in W_{2} \mid \forall v, \sum_{l=1}^{k} j_{l}^{v}=0 \text { and } \exists j_{u_{1}}^{v_{1}} \text { s.t. } \forall u_{2} \neq u_{1},\left|j_{u_{1}}^{v_{1}}\right| \neq\left|j_{u_{2}}^{v_{1}}\right|\right\} \\
\leq & Q_{k} \cdot\left(b_{N}+1\right)^{2 k-2} \cdot 2^{4 k} .
\end{aligned}
$$

where $Q_{k}=\sharp\left\{\pi=\left\{V_{1}, \ldots, V_{2 k-1}\right\} \mid \pi \in \mathcal{P}(4 k)\right\}$. When $\mathbf{J} \in W_{2}$, there are two situations. One is that $\exists i_{1}, i_{2}$ such that $\sharp S_{i_{1}}=\sharp S_{i_{2}}=3$ and for any 
$i \notin\left\{i_{1}, i_{2}\right\}, \sharp S_{i}=2$. The other situation is that $\exists i_{1}$ such that $\sharp S_{i_{1}}=4$ and for any $i \neq i_{1}, \sharp S_{i}=2$. In the first situation, suppose $S_{i_{1}}=\left\{j_{x}^{a}, j_{y}^{b}, j_{z}^{c}\right\}$, $S_{i_{2}}=\left\{j_{w}^{d}, j_{u}^{e}, j_{v}^{f}\right\}$. If $a=b=c$ and $d=e=f$, then we can find $g \in$ $\{1,2,3,4\} \backslash\{a, d\}$. Then for any $r$, there is one unique element which has the same absolute value as $j_{r}^{g}$ in $S_{\mathbf{J}}$. Then from (3.6) we know that in $\left\{j_{1}^{g}, \ldots, j_{k}^{g}\right\}$ there is at least one element which has a different absolute value from the others and then (3.7) is obtained. If $a \neq b$ and $a \neq c$, then $j_{x}^{a}$ has a different absolute value from the other elements in $\left\{j_{1}^{a}, \ldots, j_{k}^{a}\right\}$ and then (3.7) is also obtained. In the second situation, suppose $S_{i_{1}}=\left\{j_{x}^{a}, j_{y}^{b}, j_{z}^{c}, j_{w}^{d}\right\}$. If $\{a, b, c, d\} \neq\{1,2,3,4\}$ then similarly to the first situation we can get (3.7). If $\{a, b, c, d\}=\{1,2,3,4\}$, then $j_{x}^{a}$ has different absolute value from the other elements in $\left\{j_{1}^{a}, \ldots, j_{k}^{a}\right\}$ thus we also get (3.7). From the above discussion we know that for any $\mathbf{J} \in W_{2}$ such that $E\left(\prod_{v=1}^{4}\left(A\left[i^{v}, \mathbf{t}^{v}, \mathbf{j}^{v}\right]-E A\left[i^{v}, \mathbf{t}^{v}, \mathbf{j}^{v}\right]\right)\right) \neq 0$, (3.7) is satisfied. Then we get

$$
\begin{aligned}
& \Phi_{2} \\
= & \frac{1}{N^{2 k+4} m^{2 k}} E\left(\sum_{\substack{i^{1}, i^{2}, i^{3}, i^{4} \\
\mathbf{t}^{1}, \mathbf{t}^{2}, \mathbf{t}^{3}, \mathbf{t}^{4}}} \sum_{\mathbf{J} \in W_{2}} E\left(\prod_{v=1}^{4}\left(A\left[i^{v}, \mathbf{t}^{v}, \mathbf{j}^{v}\right]-E A\left[i^{v}, \mathbf{t}^{v}, \mathbf{j}^{v}\right]\right)\right)\right. \\
\leq & \frac{1}{N^{2 k+4} m^{2 k}} N^{4} m^{4 k} Q_{k}\left(b_{N}+1\right)^{2 k-2} 2^{4 k} M \\
\leq & D \cdot b_{N}^{-2}
\end{aligned}
$$

where $D$ is independent of $N$.

Evaluation of $\Phi_{3}$. When $\mathbf{J} \in W_{3}$, we have $\sharp S_{i}=2$ for all $i \in\{1, \ldots, 2 k\}$. Like the situations mentioned above, we have: $\exists u_{1} \in$ $\{1,2, \ldots, k\}$ such that $\forall u_{2} \neq u_{1},\left|j_{u_{1}}^{1}\right| \neq\left|j_{u_{2}}^{1}\right|$, then $j_{u_{1}}^{1}$ and its absolute value are determined by $j_{1}^{1}, \ldots, j_{u_{1}-1}^{1}, j_{u_{1}+1}^{1}, \ldots, j_{k}^{1}$ for $\sum_{i=1}^{k} j_{i}^{1}=0$. Suppose $\left|j_{u_{3}}^{e}\right|=\left|j_{u_{1}}^{1}\right|$ and $f \in\{1,2,3,4\} \backslash\{1, e\}$, then $\exists u_{4} \in\{1,2, \ldots, k\}$ such that $\forall u_{5} \neq u_{4},\left|j_{u_{5}}^{f}\right| \neq\left|j_{u_{4}}^{f}\right|$, then $j_{u_{4}}^{f}$ and its absolute value are determined by $j_{1}^{f}, \ldots, j_{u_{4}-1}^{f}, j_{u_{4}+1}^{f}, \ldots, j_{k}^{f}$ for $\sum_{i=1}^{k} j_{i}^{f}=0$. For $j_{u_{1}}^{1}$ and $j_{u_{4}}^{f}$ are determined, we 
have

$$
\begin{aligned}
& \sharp\left\{\mathbf{J}=\left(j_{1}^{1}, \ldots, j_{k}^{1}, \ldots, j_{k}^{4}\right) \in W_{3} \mid E\left(\prod_{v=1}^{4}\left(A\left[i^{v}, \mathbf{t}^{v}, \mathbf{j}^{v}\right]-E A\left[i^{v}, \mathbf{t}^{v}, \mathbf{j}^{v}\right]\right)\right) \neq 0\right\} \\
\leq & \left(b_{N}+1\right)^{p-2} \cdot 2^{4 k} \cdot \sharp \mathcal{P}_{2}(4 k)
\end{aligned}
$$

and thus

$$
\begin{aligned}
& \Phi_{3} \\
= & \frac{1}{N^{2 k+4} m^{2 k}} E\left(\sum_{\substack{i^{1}, i^{2}, i^{3}, i^{4} \\
\mathbf{t}^{1}, \mathbf{t}^{2}, \mathbf{t}^{\mathbf{3}^{4}}, \mathbf{t}^{4}}} \sum_{\mathbf{J} \in W_{3}} E\left(\prod_{v=1}^{4}\left(A\left[i^{v}, \mathbf{t}^{v}, \mathbf{j}^{v}\right]-E A\left[i^{v}, \mathbf{t}^{v}, \mathbf{j}^{v}\right]\right)\right)\right) \\
\leq & \frac{1}{N^{2 k+4} m^{2 k}} N^{4} m^{4 k}\left(b_{N}+1\right)^{p-2} \cdot 2^{4 k} \cdot \sharp \mathcal{P}_{2}(4 k) M \\
\leq & F \cdot b_{N}^{-2}
\end{aligned}
$$

where $F$ is independent of $N$. Finally,

$$
\frac{1}{N^{4}} E\left(\left(\operatorname{tr} X_{N}^{k}-E\left(\operatorname{tr} X_{N}^{k}\right)\right)^{4}\right)=\Phi_{1}+\Phi_{2}+\Phi_{3} \leq(C+D+F) \cdot b_{N}^{-2} .
$$

For $b_{N}=N-1,(3.4)$ is proved and we have proved the theorem.

Remark 3.3 (Hankel block matrices and complex Toeplitz case). If $H_{N}=\left(A_{N+1-i-j}\right)$ is a block Hankel matrix which is defined as in the introduction, then the eigenvalue distribution of $H_{N} / \sqrt{m N}$ converges almost surely to a distribution $\gamma_{H}^{(m)}$ which is determined by its even moments

$$
m_{2 k}\left(\gamma_{H}^{(m)}\right)=\sum_{\pi \in \mathcal{P}_{2}^{1}(2 k)} \frac{r(m, \pi)}{m^{k+1}} \int_{[0,1] \times[-1,1]^{k}} \prod_{j=1}^{2 k} I_{[0,1]}\left(x_{0}-\sum_{q=1}^{j}(-1)^{q} x_{\pi(q)}\right) \prod_{l=0}^{k} \mathrm{~d} x_{l}
$$

where $r(m, \pi)=\sharp\left\{\left(t_{1}, . ., t_{2 k}\right) \mid E\left(a_{t_{1} t_{2}}\left(j_{1}\right), \ldots, a_{t_{2 k} t_{1}}\left(j_{2 k}\right)\right) \neq 0\right\}$.

Suppose $\pi=\left\{\left\{a_{1}, b_{1}\right\}, \ldots,\left\{a_{k}, b_{k}\right\}\right\}$, then

$$
\begin{aligned}
& r(m, \pi) \\
= & \sharp\left\{\left(t_{1}, . ., t_{2 k}\right) \mid 1 \leq t_{a_{i}}, t_{b_{i}} \leq m ;\left\{\begin{array}{l}
t_{a_{i}}=t_{b_{i}} \\
t_{a_{i+1}}=t_{b_{i+1}}
\end{array} \text { or }\left\{\begin{array}{l}
t_{a_{i}}=t_{b_{i+1}} \\
t_{a_{i+1}}=t_{b_{i}}
\end{array} \quad(1 \leq i \leq k)\right\}\right.\right. \\
= & \sharp\left\{\left(t_{1}, . ., t_{2 k}\right) \mid 1 \leq t_{a_{i}}, t_{b_{i}} \leq m ; t_{a_{i}}=t_{\left(b_{i}+1\right)} ; t_{\left(a_{i}+1\right)}=t_{b_{i}}(1 \leq i \leq k)\right\} \\
+ & O\left(m^{k}\right) \\
= & m^{2 k-f(\pi)}+O\left(m^{k}\right)
\end{aligned}
$$


If the blocks of $H_{N}$ are Hermitian matrices, the results would be similar. If the blocks in a block Toeplitz matrix $T=\left(A_{i-j}\right)_{i, j=1}^{N}$ are complex matrices and $A_{-s}=\left(\overline{A_{s}}\right)^{T}$, then the results would also be similar.

Remark 3.4 (relation with dynamical system). $f(\pi)$ has a relation with dynamical system. Let $\pi=\left\{V_{1}, V_{2}, \ldots, V_{k}\right\}, V_{r}=\left\{a_{r}, b_{r}\right\}(1 \leq r \leq k)$, then $f(\pi)$ is the number of linearly independent equations of (3.1). Now we consider a discrete dynamical system. Give $[2 k]=\{1, \ldots, 2 k\}$ the discrete topology. Let $\phi$ be a self-homeomorphism on $[2 k]$ and $\phi\left(a_{i}\right)=b_{i}+1, \phi\left(b_{i}\right)=$ $a_{i}+1(1 \leq i \leq k)$. Consider a continuous map $\psi: \mathbb{Z} \times[2 k] \rightarrow[2 k]$ such that $\psi(s, t)=\phi^{s}(t)(s \in \mathbb{Z}, t \in[2 k])$ then $\psi$ becomes a dynamical system. Obviously we see that the number of orbits of this dynamical system equals the number of independent variables in (3.1) and then equals $2 k-f(\pi)$.

\section{Block Toeplitz and Hankel Band Matrices}

Definition 4.1. Let $T_{N}=\left(A_{i-j}\right)_{i, j=1}^{N}$ be an $m N \times m N$ block Toeplitz matrix. We call $T$ a block Toeplitz band matrix if $\exists b_{N}<N$ s.t. $A_{s}=0$ when $|s|>b_{N}$. We call $b_{N}$ the bandwidth of the matrix.

Theorem 4.2 (proportional growth). Let $T_{N}$ be a block Toeplitz band matrix with the bandwidth $b_{N} \sim b N, b \in(0,1]$. Take the normalization

$$
X_{N}=T_{N} / \sqrt{m(2-b) b N} .
$$

With the notation and assumptions of Theorem 3.2, $\mu_{X_{N}}$ converges almost surely to a symmetric probability distribution $\gamma_{T}^{(m)}(b)$ which is determined by its even moments

$m_{2 k}\left(\gamma_{T}^{(m)}(b)\right)=\frac{1}{(2-b)^{k}} \sum_{\pi \in \mathcal{P}_{2}(2 k)} m^{k-1-f(\pi)} \int_{[0,1] \times[-1,1]^{k}} \prod_{j=1}^{2 k} I_{[0,1]}\left(x_{0}+b \sum_{i=1}^{j} \epsilon_{\pi}(i) x_{\pi(i)}\right) \prod_{l=0}^{k} \mathrm{~d} x_{l}$

where $\epsilon_{\pi}(i)=1$ if $i$ is the smaller number of $V_{\pi(i)}$ and $\epsilon_{\pi}(i)=-1$ otherwise.

Theorem 4.3 (slow growth). Let $T_{N}$ be a block Toeplitz band matrices with the bandwidth $b_{N}=o(N)$ but $b_{N} \rightarrow \infty$. Take the normalization

$$
X_{N}=T_{N} /\left(\sqrt{2 m b_{N}}\right)
$$


With the notation and assumptions of Theorem 3.2, $\mu_{X_{N}}$ converges weakly to a distribution $\gamma^{(m)}$ which is determined by its even moments

$$
m_{2 k}\left(\gamma^{(m)}\right)=\sum_{\pi \in \mathcal{P}_{2}(2 k)} m^{k-1-f(\pi)} .
$$

In addition, if there exist positive constants $\epsilon_{0}$ and $C$ such that

$$
b_{N} \geq C \cdot N^{\frac{1}{2}+\epsilon_{0}},
$$

then $\mu_{X_{N}}$ converges almost surely to $\gamma^{(m)}$.

Proof of Theorem 4.2. It is easy to see that Lemma 2.2 is also right for block

Toeplitz band matrices but now $b_{N}$ is no longer $N-1$. Let

$$
m_{k, N}=\int x^{k} \mathrm{~d} \mu_{X_{N}}=\frac{1}{m N} E\left(\operatorname{tr} X_{N}^{k}\right)=\frac{1}{m N}(m(2-b) b N)^{-\frac{k}{2}} E\left(\operatorname{tr} T_{N}^{k}\right) .
$$

Using Lemma 2.2, we get

$$
\begin{aligned}
& \frac{1}{m_{k, N}} \frac{1}{(2-b)^{\frac{k}{2}}} \frac{m^{N}}{m} \sum_{i=1}^{N} \sum_{j_{1}, \ldots, j_{k}=-b_{N}}^{b_{N}} \sum_{t_{1}, \ldots, t_{k}=1}^{m} \frac{E\left(a_{t_{1} t_{2}}\left(j_{1}\right) \cdots a_{t_{k} t_{1}}\left(j_{k}\right)\right)}{(m b N)^{\frac{k}{2}}} \prod_{l=1}^{k} I_{[1, N]}\left(i+\sum_{q=1}^{l} j_{q}\right) \delta_{0, \sum_{q=1}^{k} j_{q}} \\
= & \frac{1}{(2-b)^{\frac{k}{2}}}\left(\frac{1}{b}\right)^{\frac{k}{2}} \sum_{i=1}^{N} \sum_{j_{1}, \ldots, j_{k}=-b_{N}}^{b_{N}} \sum_{t_{1}, \ldots, t_{k}=1}^{m} \frac{E\left(a_{t_{1} t_{2}}\left(j_{1}\right) \cdots a_{t_{k} t_{1}}\left(j_{k}\right)\right)}{(m N)^{\frac{k}{2}+1}} \prod_{l=1}^{k} I_{[1, N]}\left(i+\sum_{q=1}^{l} j_{q}\right) \delta_{0, \sum_{q=1}^{k} j_{q}} .
\end{aligned}
$$

Similarly as in the proof of Theorem 3.2, we know that when $k$ is odd,

$$
\sum_{i=1}^{N} \sum_{j_{1}, \ldots, j_{k}=-b_{N}}^{b_{N}} \sum_{t_{1}, \ldots, t_{k}=1}^{m} \frac{E\left(a_{t_{1} t_{2}}\left(j_{1}\right) \cdots a_{t_{k} t_{1}}\left(j_{k}\right)\right)}{(m N)^{\frac{k}{2}+1}} \prod_{l=1}^{k} I_{[1, N]}\left(i+\sum_{q=1}^{l} j_{q}\right) \delta_{0, \sum_{q=1}^{k} j_{q}}=o(1)
$$

and then $m_{k, N}=o(1)$.

For $m_{2 k, N}$,

$$
\begin{aligned}
& \sum_{i=1}^{N} \sum_{j_{1}, \ldots, j_{2 k}=-b_{N}}^{b_{N}} \sum_{t_{1}, \ldots, t_{2 k}=1}^{m} \frac{E\left(a_{t_{1} t_{2}}\left(j_{1}\right) \cdots a_{t_{2 k} t_{1}}\left(j_{2 k}\right)\right)}{(m N)^{k+1}} \prod_{l=1}^{2 k} I_{[1, N]}\left(i+\sum_{q=1}^{l} j_{q}\right) \delta_{0, \sum_{q=1}^{2 k} j_{q}} \\
= & N^{-k-1} \sum_{i=1}^{N} \sum_{\pi \in \mathcal{P}_{2}(2 k)} \sum_{x_{1}, \ldots, x_{k}=-b_{N}}^{b_{N}} m^{k-1-f(\pi)} \prod_{l=1}^{2 k} I_{[1, N]}\left(i+\sum_{q=1}^{l} \epsilon_{\pi}(q) x_{\pi(q)}\right)+o(1) .
\end{aligned}
$$


So

$$
=\frac{1}{(2-b)^{k}}\left(\frac{1}{b}\right)^{k} N^{-k-1} \sum_{i=1}^{N} \sum_{\pi \in \mathcal{P}_{2}(2 k)} \sum_{x_{1}, \ldots, x_{k}=-b_{N}}^{b_{N}} m^{k-1-f(\pi)} \prod_{l=1}^{2 k} I_{[1, N]}\left(i+\sum_{q=1}^{l} \epsilon_{\pi}(q) x_{\pi(q)}\right)+o(1),
$$

thus

$$
\lim _{N \rightarrow \infty} m_{2 k, N}=\frac{1}{(2-b)^{k}} \sum_{\pi \in \mathcal{P}_{2}(2 k)} m^{k-1-f(\pi)} \int_{[0,1] \times[-1,1]} \prod_{j=1}^{2 k} I_{[0,1]}\left(x_{0}+b \sum_{q=1}^{j} \epsilon_{\pi}(q) x_{\pi(q)}\right) \prod_{l=0}^{k} \mathrm{~d} x_{l} .
$$

Let $m_{2 k}=\lim _{N \rightarrow \infty} m_{2 k, N}$ and $m_{2 k-1}=0(k \in \mathbb{N})$, then for any $k \in \mathbb{N}$ we have $\lim _{N \rightarrow \infty} m_{k, N}=m_{k}$. It is easy to see that

$$
m_{2 k} \leq \sum_{\pi \in \mathcal{P}_{2}(2 k)} m^{k-1-f(\pi)} \leq(2 k-1) ! ! \cdot m^{k-1}
$$

and then using Carleman's theorem (see [6]) we know that the limit distribution $\gamma_{T}^{(m)}(b)$ is determined by its even moments $\left\{m_{2 k}\right\}$. Similar to the proof of Theorem 3.2 we can easily prove that $\mu_{X_{N}}$ converges almost surely to $\gamma_{T}^{(m)}(b)$. Then Theorem 4.2 is proved.

Proof of Theorem 4.3. Let

$$
\begin{aligned}
& m_{k, N} \\
= & \int x^{k} \mathrm{~d} \mu_{X_{N}}=\frac{1}{m N} E\left(\operatorname{tr} X_{N}^{k}\right)=\frac{1}{m N}\left(2 m b_{N}\right)^{-\frac{k}{2}} E\left(\operatorname{tr} T_{N}^{k}\right) \\
= & \frac{(m N)^{\frac{k}{2}+1}}{m N\left(2 m b_{N}\right)^{\frac{k}{2}}} \sum_{i=1}^{N} \sum_{j_{1}, \ldots, j_{k}=-b_{N}}^{b_{N}} \sum_{t_{1}, \ldots, t_{k}=1}^{m} \frac{E\left(a_{t_{1} t_{2}}\left(j_{1}\right) \cdots a_{t_{k} t_{1}}\left(j_{k}\right)\right)}{(m N)^{\frac{k}{2}+1}} \prod_{l=1}^{k} I_{[1, N]}\left(i+\sum_{q=1}^{l} j_{q}\right) \delta_{0, \sum_{q=1}^{k} j_{q}} .
\end{aligned}
$$

Similarly as in the proof of Theorem 3.2, we know that when $k$ is odd, $m_{k, N}=o(1)$. For $m_{2 k, N}$,

$$
\begin{aligned}
& \sum_{i=1}^{N} \sum_{j_{1}, \ldots, j_{2 k}=-b_{N}}^{b_{N}} \sum_{t_{1}, \ldots, t_{2 k}=1}^{m} \frac{E\left(a_{t_{1} t_{2}}\left(j_{1}\right) \cdots a_{t_{2 k} t_{1}}\left(j_{2 k}\right)\right)}{(m N)^{k+1}} \prod_{l=1}^{2 k} I_{[1, N]}\left(i+\sum_{q=1}^{l} j_{q}\right) \delta_{0, \sum_{q=1}^{2 k} j_{q}} \\
= & N^{-k-1} \sum_{i=1}^{N} \sum_{\pi \in \mathcal{P}_{2}(2 k)} \sum_{x_{1}, \ldots, x_{k}=-b_{N}}^{b_{N}} m^{k-1-f(\pi)} \prod_{l=1}^{2 k} I_{[1, N]}\left(i+\sum_{q=1}^{l} \epsilon_{\pi}(q) x_{\pi(q)}\right)+o(1) .
\end{aligned}
$$


So

$$
\begin{aligned}
& m_{k, N} \\
= & \frac{m^{k+1}}{m N\left(2 m b_{N}\right)^{k}} \sum_{i=1}^{N} \sum_{\pi \in \mathcal{P}_{2}(2 k)} \sum_{x_{1}, \ldots, x_{k}=-b_{N}}^{b_{N}} m^{k-1-f(\pi)} \prod_{l=1}^{2 k} I_{[1, N]}\left(i+\sum_{q=1}^{l} \epsilon_{\pi}(q) x_{\pi(q)}\right)+o(1) \\
= & \frac{1}{N \cdot 2^{k} \cdot b_{N}^{k}} \sum_{i=1}^{N} \sum_{\pi \in \mathcal{P}_{2}(2 k)} \sum_{x_{1}, \ldots, x_{k}=-b_{N}}^{b_{N}} m^{k-1-f(\pi)} \prod_{l=1}^{2 k} I_{[1, N]}\left(i+\sum_{q=1}^{l} \epsilon_{\pi}(q) x_{\pi(q)}\right)+o(1) .
\end{aligned}
$$

Thus

$$
\begin{aligned}
\lim _{N \rightarrow \infty} m_{2 k, N} & =\frac{1}{2^{k}} \sum_{\pi \in \mathcal{P}_{2}(2 k)} m^{k-1-f(\pi)} \int_{[0,1] \times[-1,1]^{k}} \prod_{l=0}^{k} \mathrm{~d} x_{l} \\
& =\sum_{\pi \in \mathcal{P}_{2}(2 k)} m^{k-1-f(\pi)} .
\end{aligned}
$$

Let $m_{2 k}=\lim _{N \rightarrow \infty} m_{2 k, N}$ and $m_{2 k-1}=0(k \in \mathbb{N})$, then for any $k \in \mathbb{N}$ we have $\lim _{N \rightarrow \infty} m_{k, N}=m_{k}$. It is easy to see that

$$
m_{2 k} \leq \sum_{\pi \in \mathcal{P}_{2}(2 k)} m^{k-1-f(\pi)} \leq(2 k-1) ! ! \cdot m^{k-1}
$$

and then using Carleman's theorem (see [6]) we know that $\mu_{X_{N}}$ converges weakly to a distribution $\gamma^{(m)}$ which is determined by its even moments $\left\{m_{2 k}\right\}$. Similarly as in the the proof of Theorem 3.2 we know that

$$
\frac{1}{N^{4}} E\left(\left(\operatorname{tr} X_{N}^{k}-E\left(\operatorname{tr} X_{N}^{k}\right)\right)^{4}\right) \leq B \cdot b_{N}^{-2}
$$

where $B$ is a constant and is independent of $N$. For $b_{N} \geq C \cdot N^{\frac{1}{2}+\epsilon_{0}}$, we have

$$
\frac{1}{N^{4}} E\left(\left(\operatorname{tr} X_{N}^{k}-E\left(\operatorname{tr} X_{N}^{k}\right)\right)^{4}\right) \leq B \cdot C^{-2} \cdot N^{-1-2 \epsilon_{0}} .
$$

Therefore

$$
\sum_{N=1}^{\infty} \frac{1}{N^{4}} E\left(\left(\operatorname{tr} X_{N}^{k}-E\left(\operatorname{tr} X_{N}^{k}\right)\right)^{4}\right)<\infty
$$

and then $\mu_{X_{N}}$ converges almost surely to the limit distribution $\gamma^{(m)}$. 
Remark 4.4. In [15], the authors proved that the limit of eigenvalue distribution for band Toeplitz random matrix with bandwidth $b_{N}=o(N)$ is the standard normal distribution $\mathrm{N}(0,1)$. But in this paper, the matrix is block Toeplitz and the conclusion will be different. The expectation of $\gamma^{(m)}$ is 0 and the variance of $\gamma^{(m)}$ is $\sum_{\pi \in \mathcal{P}_{2}(2)} m^{-f(\pi)}=1$. The forth moment of $\gamma^{(m)}$ is $\sum_{\pi \in \mathcal{P}_{2}(4)} m^{1-f(\pi)}=2+\frac{1}{m^{2}}$ which is not the forth moment of $\mathrm{N}(0,1)$ if $m \neq 1$. So $\gamma^{(m)}$ is not $\mathrm{N}(0,1)$ when $m \neq 1$.

\section{Convergence to Semicircle Law}

Suppose $\pi=\left\{\left\{a_{1}, b_{1}\right\}, \ldots,\left\{a_{k}, b_{k}\right\}\right\} \in \mathcal{P}_{2}(2 k)$, the system of linear equations determined by $\pi$ is (see Definition 3.1)

$$
\begin{cases}t_{a_{i}}=t_{b_{i}+1} & 1 \leq i \leq k \\ t_{b_{i}}=t_{a_{i}+1} & 1 \leq i \leq k\end{cases}
$$

where $t_{2 k+1}=t_{1}$. This system can be rewritten as

$$
\left\{\begin{array}{l}
t_{s_{1}(1)}=t_{s_{1}(2)}=\cdots=t_{s_{1}\left(r_{1}\right)}=t_{s_{1}(1)} \\
\cdots \\
t_{s_{p}(1)}=t_{s_{p}(2)}=\cdots=t_{s_{p}\left(r_{p}\right)}=t_{s_{p}(1)}
\end{array}\right.
$$

such that $\left\{s_{1}(1), \ldots, s_{1}\left(r_{1}\right)\right\} \cup \cdots \cup\left\{s_{p}(1), \ldots, s_{p}\left(r_{p}\right)\right\}=\{1,2, \ldots, 2 k\}$ and $\left\{s_{i}(1), \ldots, s_{i}\left(r_{i}\right)\right\} \cap\left\{s_{j}(1), \ldots, s_{j}\left(r_{j}\right)\right\}=\emptyset$ if $i \neq j$. We call $t_{s_{i}(1)}=t_{s_{i}(2)}=$ $\cdots=t_{s_{i}\left(r_{i}\right)}=t_{s_{i}(1)}$ a circle of $\pi$. We use $g(\pi)$ to denote the number of the circles of $\pi$. For example, if $\pi=\{\{1,2\},\{3,4\},\{5,6\},\{7,8\}\} \in \mathcal{P}_{2}(8)$, then the system of linear equations determined by $\pi$ is

$$
\left\{\begin{array}{l}
t_{1}=t_{3} \\
t_{2}=t_{2} \\
t_{3}=t_{5} \\
t_{4}=t_{4} \\
t_{5}=t_{7} \\
t_{6}=t_{6} \\
t_{7}=t_{1} \\
t_{8}=t_{8}
\end{array}\right.
$$


This system can be rewritten as

$$
\left\{\begin{array}{l}
t_{1}=t_{3}=t_{5}=t_{7}=t_{1} \\
t_{2}=t_{2} \\
t_{4}=t_{4} \\
t_{6}=t_{6} \\
t_{8}=t_{8}
\end{array} .\right.
$$

So this $\pi$ has five circles: $t_{1}=t_{3}=t_{5}=t_{7}=t_{1}, t_{2}=t_{2}, t_{4}=t_{4}, t_{6}=t_{6}$ and $t_{8}=t_{8}$, thus $g(\pi)=5$. These circles can be denoted by
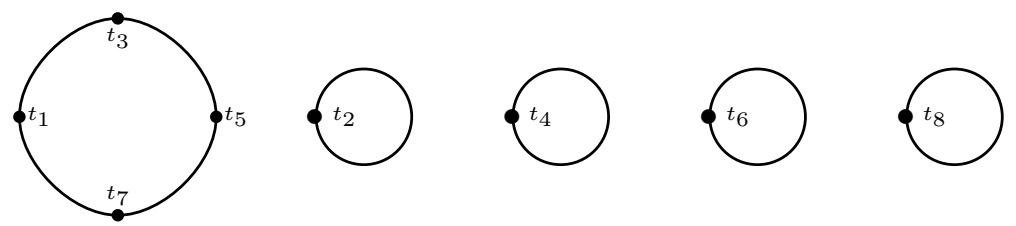

For $f(\pi)$ denotes the number of independent equations of the system, it is easy to see that $g(\pi)=2 k-f(\pi)$ for any $\pi \in \mathcal{P}_{2}(2 k)$.

Lemma 5.1. For any $\pi \in \mathcal{P}_{2}(2 k), g(\pi) \leq k+1$.

The proof of Lemma 5.1 can be found in [22].

We now review the concept of noncrossing partition (see [21]). A partition $\pi \in \mathcal{P}(n)$ is called noncrossing if whenever four elements $1 \leq a<b<$ $c<d \leq n$ are such that $a \sim_{\pi} c$ and $b \sim_{\pi} d$, then $a \sim_{\pi} b \sim_{\pi} c \sim_{\pi} d$.

Lemma 5.2. For any $\pi \in \mathcal{P}_{2}(2 k), g(\pi)=k+1$ if and only if $\pi$ is noncrossing.

The proof of Lemma 5.2 can be found in [20].

Theorem 5.3. Suppose $\gamma_{T}^{(m)}$ is defined as in Theorem 3.2. As $m \rightarrow \infty$, $\gamma_{T}^{(m)}$ converges weakly to the semicircle law $w(x)$, i.e.,

$$
w(x)= \begin{cases}\frac{1}{2 \pi} \sqrt{4-x^{2}} & |x| \leq 2 \\ 0 & |x|>2\end{cases}
$$

Proof of Theorem 5.3. From Theorem 3.2 we know that the odd moments of $\gamma_{T}^{(m)}$ are all zero and its even moments are

$m_{2 k}\left(\gamma_{T}^{(m)}\right)=\sum_{\pi \in \mathcal{P}_{2}(2 k)} m^{k-1-f(\pi)} \int_{[0,1] \times[-1,1]^{k}} \prod_{j=1}^{2 k} I_{[0,1]}\left(x_{0}+\sum_{q=1}^{j} \epsilon_{\pi}(q) x_{\pi(q)}\right) \prod_{l=0}^{k} \mathrm{~d} x_{l}$ 
where

$$
\epsilon_{\pi}(q)= \begin{cases}1 & \text { if } q \text { is the smaller element of } V_{\pi(q)}, \\ -1 & \text { if } q \text { is the larger element of } V_{\pi(q)} .\end{cases}
$$

From Lemma 5.1 and Lemma 5.2 we know that if $\pi \in \mathcal{P}_{2}(2 k)$, then $f(\pi)=k-1$ if $\pi$ is noncrossing, otherwise $f(\pi)>k-1$. So we have

$$
\lim _{m \rightarrow \infty} m_{2 k}\left(\gamma_{T}^{(m)}\right)=\sum_{\substack{\pi \in \mathcal{P}_{2}(2 k) \\ \pi \text { is noncrossing }}} \int_{[0,1] \times[-1,1]} \prod_{j=1}^{2 k} I_{[0,1]}\left(x_{0}+\sum_{q=1}^{j} \epsilon_{\pi}(q) x_{\pi(q)}\right) \prod_{l=0}^{k} \mathrm{~d} x_{l} .
$$

From [4] we know that when $\pi$ is noncrossing,

$$
\int_{[0,1] \times[-1,1]^{k}} \prod_{j=1}^{2 k} I_{[0,1]}\left(x_{0}+\sum_{q=1}^{j} \epsilon_{\pi}(q) x_{\pi(q)}\right) \prod_{l=0}^{k} \mathrm{~d} x_{l}=1 .
$$

So

$$
\lim _{m \rightarrow \infty} m_{2 k}\left(\gamma_{T}^{(m)}\right)=\sum_{\substack{\pi \in \mathcal{P}_{2}(2 k) \\ \pi \text { is noncrossing }}} 1 .
$$

From [11] we know that

$$
\sharp\left\{\pi \in \mathcal{P}_{2}(2 k) \mid \pi \text { is noncrossing }\right\}=C_{k}
$$

where $C_{k}$ is Catalan number. So

$$
\lim _{m \rightarrow \infty} m_{2 k}\left(\gamma_{T}^{(m)}\right)=C_{k} .
$$

For $C_{k}$ is the $2 k$-th moment of $w(x)$ whose odd moments are all zero, we know that $\gamma_{T}^{(m)}$ converges weakly to $w(x)$ thus the theorem is proved.

Remark 5.4. If the assumptions in Theorem 4.3 are all satisfied, then it is easy to see that as $m \rightarrow \infty, \gamma^{(m)}$ converges weakly to the semicircle law $w(x)$.

Remark 5.5. For a block Hankel matrix as we discussed in Remark 3.3 we also have that as $m \rightarrow \infty, \gamma_{H}^{(m)}$ converges weakly to the semicircle law $w(x)$ because (see (3.9))

$$
\begin{aligned}
r(m, \pi) & =\sharp\left\{\left(t_{1}, . ., t_{2 k}\right) \mid 1 \leq t_{a_{i}}, t_{b_{i}} \leq m ; t_{a_{i}}=t_{\left(b_{i}+1\right)} ; t_{\left(a_{i}+1\right)}=t_{b_{i}}(1 \leq i \leq k)\right\} \\
& +O\left(m^{k}\right) \\
& =m^{2 k-f(\pi)}+O\left(m^{k}\right)
\end{aligned}
$$


and $\left\{\pi \in \mathcal{P}_{2}(2 k) \mid \pi\right.$ is noncrossing $\} \subset \mathcal{P}_{2}^{1}(2 k)$ and the fact that the integral in (3.8) is 1 when $\pi$ is noncrossing (see [4]). If the blocks of $H_{N}$ are Hermitian matrices, the results would be similar. If the blocks in a block Toeplitz matrix $T=\left(A_{i-j}\right)_{i, j=1}^{N}$ are complex matrices and $A_{-s}=\left(\overline{A_{s}}\right)^{T}$, then the results would also be similar.

Remark 5.6. If the blocks in a block Toeplitz matrix $T=\left(A_{i-j}\right)_{i, j=1}^{N}$ are symmetric matrices and $A_{-s}=A_{s}$, just like those discussed in [18], then the eigenvalue distribution of $T / \sqrt{m N}$ converges almost surely to a distribution ${\widetilde{\gamma_{T}}}^{(m)}$ which is determined by its even moments

$m_{2 k}\left({\widetilde{\gamma_{T}}}^{(m)}\right)=\sum_{\pi \in \mathcal{P}_{2}(2 k)} \frac{r(m, \pi)}{m^{k+1}} \int_{[0,1] \times[-1,1] k} \prod_{j=1}^{2 k} I_{[0,1]}\left(x_{0}+\sum_{q=1}^{j} \epsilon_{\pi}(q) x_{\pi(q)}\right) \prod_{l=0}^{k} \mathrm{~d} x_{l}$

where $r(m, \pi)$ is the same as (3.9). So we also have that as $m \rightarrow \infty, \widetilde{\gamma_{T}}{ }^{(m)}$ converges weakly to the semicircle law $w(x)$.

\section{Acknowledgements}

The authors thank the anonymous referee for his heuristic question: what is the iterated limit $\lim _{m \rightarrow \infty} \lim _{N \rightarrow \infty} \mu_{X_{N}}$. This question leads to the semicircle law we discussed in Section 5 .

\section{References}

[1] Z. D. Bai, Methodologies in spectral analysis of large-dimensional random matrices, a review, Statist. Sinica. 9 (1999), 611-677.

[2] A. Basak, and A. Bose, Limiting spectral distribution of some band matrices, Unpublished manuscript (2009), http://www.isical.ac.in/ statmath/html/publication/bandpaper.pdf.

[3] L. V. Bogachev, S. A. Molchanov and L. A. Pastur, On the level density of random band matrix, Math. Notes. 50 (1991), 1232-1242.

[4] A. Bose and A. Sen, Another look at the moment method for large dimensional random matrices, Electron. J. Probab. 13 (2008), 588-628.

[5] W. Bryc, A. Dembo and T. Jiang, Spectral measure of large random Hankel, Markov and Toeplitz matrices, Ann. Probab. 34 (2006), no.1, $1-38$.

[6] W. Feller, An Introduction to Probability Theory and Its Applications, Volum 2, Wiley, New York, 1971. 
[7] H. Gazzah, P. A. Regalia and J. P. Delmas, Asymptotic eigenvalue distribution of block Toeplitz matrices and application to blind SIMO channel identification, IEEE Trans. Inf. Theory. 47 (2001), 1243-1251.

[8] U. Grenander and G. Szegö, Toeplitz forms and their applications, 2nd ed., Chelsea, New York, 1984.

[9] C. Hammond and S. J. Miller, Distribution of eigenvalues for the ensemble of real symmetric Toeplitz matrices, J. Theoret. Probab. 18 (2005), $537-566$.

[10] J. W. Hardy, Adaptive optics, Sci.Am. 270(1994), 60-65.

[11] F. Hiai and D. Petz, The semicircle law, free random variables and entropy, American Mathematical Society, Providence, 2000.

[12] P. Horn and C. Johnson, Topics in matrix analysis, Cambridge University Press, Cambridge, 1986.

[13] V. Kargin, Spectrum of random Toeplitz matrices with band structure, Electron. Commun. Probab. 14 (2009), 412-423.

[14] H. Khalil, B. Mourrain and M. Schatzman, Toeplitz and Toeplitz-blockToeplitz matrices and their correlation with syzygies of polynomials, Numer. Linear Algebr. Appl. 14 (2007), 581-602.

[15] D. Z. Liu and Z. D. Wang, Limit distribution of eigenvalues for random Hankel and Toeplitz band matrices, J. Theoret. Probab., 10.1007/s10959-009-0260-4 (2009).

[16] M. L. Mehta, Random Matrices, 3rd ed., Academic Press, San Diego, 2004.

[17] T. Oraby, The spectral laws of Hermitian block-matrices with large random blocks, Electron. Commun. Probab. 12 (2007), 465-476.

[18] R. Rashidi Far, T. Oraby, W. Bryc and R. Speicher, Spectra of large block matrices, Unpublished manuscript (2006), http://xxx.lanl.gov/abs/cs.IT/0610045.

[19] F. Sebert, L. Ying and Y. M. Zhou, Toeplitz block matrices in compressed sensing, Unpublished manuscript (2006), arXiv:0803.0755v1.

[20] D. Shlyakhtenko, Notes on free probability theory, Unpublished manuscript (2005), arXiv:math/0504063v1.

[21] R. Simion, Noncrossing partitions, Discret. Math. 217 (2000), 367-409.

[22] D. Voiculescu, Limit laws for random matrices and free products, Invent. Math. 104 (1991), 201-220.

[23] E. P. Wigner, Characteristic vectors of bordered matrices with infinite dimensions, Ann. Math. 12 (1955), 548-564.

[24] E. P. Wigner, On the distribution of the roots of certain symmetric matrices, Ann. Math. 67 (1958), 325-327. 